\title{
Reusabilidade em SOA: Um Mapeamento Sistemático da Literatura
}

\author{
Joyce Aline Oliveira ${ }^{1}$, Filipe G. Ramos ${ }^{2}$, Jose Jorge L. Dias Junior ${ }^{1,2}$ \\ ${ }^{1}$ Centro de Informática - Universidade Federal de Pernambuco (UFPE) \\ ${ }^{2}$ Departamento de Ciências Exatas - Universidade Federal da Paraíba (UFPB) \\ japo@cin.ufpe.br, \{filipe.guimaraes, jorge\}@dce.ufpb.br
}

\begin{abstract}
Regarding the challenges and benefits related to the SOA reuse, this paper presents a Systematic Literature Mapping about this attribute. It aims at identifying factors that influences the reusability, strategies adopted and metrics related to reuse in a SOA context. The 22 mapped papers have evidenced that cost is the most driver to reuse adoption. The strategy most adopted is Software Product Line and the service analysis and design activities are used widely. This paper is relevant to the researchers and practitioners that need a structured work about this area.
\end{abstract}

Resumo. Considerando os desafios e os beneficios inerentes ao reuso em SOA, o artigo apresenta um Mapeamento Sistemático da Literatura sobre este atributo com o propósito de identificar fatores que influenciam a reusabilidade, estratégias adotadas, fases do ciclo de vida e métricas associadas ao reuso em um contexto SOA. Os 22 artigos mapeados evidenciaram que o maior influenciador do reuso é o custo, a estratégia mais adotada é Linha de Produtos de Software e as fases de análise e de projeto de serviços são amplamente abordadas. A pesquisa é relevante para pesquisadores e organizações que busquem um estudo estruturado sobre o tema.

\section{Introdução}

A Arquitetura Orientada a Serviços (SOA) tem sido amplamente utilizada pelas organizações como uma estratégia no alcance da flexibilidade, da integração e da diminuição de custos do desenvolvimento de softwares.

A adoção de SOA possibilita que funcionalidades sejam utilizadas como serviços na construção de novas aplicações sem a necessidade de reimplementá-las [Josutis, 2007]. A estratégia da reusabilidade em SOA consiste em utilizar os serviços não apenas em um único sistema, mas em um grupo deles para aumentar a agilidade de desenvolvimento [Alferez e Pelechano 2011]. Portanto, a reutilização é um dos grandes benefícios prometidos ao se adotar SOA.

De acordo com Karthikeyan e Geetha (2013), os serviços reutilizáveis aumentam o retorno do investimento (ROI) e também reduzem custos relacionados a projeto, desenvolvimento, teste e manutenção de softwares. A reusabilidade também é tida como um dos principais critérios para a avaliação da qualidade de serviços. Outro benefício citado por Karthikeyan e Geetha (2013) é a capacidade de criar novos processos de 
negócio a partir de serviços existentes para atender as necessidades dos clientes e as exigências do mercado.

Modelos de maturidade em SOA propostos apresentam reusabilidade como um atributo que deve ser internalizado na organização a fim de alcançá-lo [Dias et al., 2012]. Além disso, estudos de caso mostram que reusabilidade é um dos principais benefícios pretendidos ao se adotar SOA [Dias et al., 2013].

Devido às vantagens mencionadas, a busca pelo reuso tem sido um dos motivos para a ampla adoção de SOA, visto ser este um dos princípios de projetos orientados a serviços [Shanmugasundaram et al., 2012] [Erl, 2005]. No entanto, apesar dos benefícios apresentados, a dificuldade em definir quais serviços reutilizar e qual processo adotar para construir serviços reutilizáveis tem sido reportada em pesquisas empíricas no âmbito de SOA [Brahe, 2007].

De acordo com Shanmugasundaram et al. (2012) muitas pesquisas focam qualidades de atributos de SOA, mas não exploram especificamente o atributo de reusabilidade. A investigação da veracidade desta premissa, os benefícios propostos pela reusabilidade e as dificuldades relatadas empiricamente inerentes ao processo de reuso motivaram a realização de um Mapeamento Sistemático da Literatura com o propósito de identificar: o estado da arte neste contexto; os fatores que influenciam positivamente e negativamente a reusabilidade, as estratégias comumente adotadas para a melhoria contínua do reuso, as métricas associadas a mensuração desse atributo, entre outros aspectos.

A pesquisa é academicamente relevante por não ter sido encontrado, até o estudado, um trabalho semelhante que agregue diferentes aspectos da reusabilidade em SOA através de um Mapeamento Sistemático. Em nível prático, a pesquisa pode ser útil para profissionais e interessados em SOA que visam compreender as características associadas à reusabilidade e não contam com uma revisão estruturada sobre o tema.

A seção 2 descreve o método utilizado para conduzir a pesquisa. A seção 3 apresenta os resultados, a seção 4 discute os resultados obtidos. A seção 5 conclui trabalho apresentando as limitações, as contribuições da pesquisa e trabalhos futuros.

\section{Método de Pesquisa}

\subsection{Questões de Pesquisa}

Para guiar a execução do mapeamento, foram definidas algumas questões de pesquisa. O objetivo, ao final da pesquisa, é responder estas questões:

QP1. Quais são os fatores que influenciam a reusabilidade em SOA?

Objetivo: identificar os fatores que tem alguma influência positiva ou negativa na reusabilidade, nos serviços ou em SOA como um todo.

QP2. Quais são as estratégias adotadas para melhorar a reusabilidade em SOA?

Objetivo: descobrir as estratégias adotadas para tentar potencializar o reuso em SOA. Por exemplo: utilizar registro de serviços, utilizar técnica de linha de produto, utilizar métricas de avaliação, etc.

QP3. Qual fase do ciclo de vida de desenvolvimento a estratégia é adotada? 
Objetivo: identificar em que fase ou ciclo de vida do serviço ou na SOA, onde a estratégia é aplicada. Por exemplo: na fase de requisitos, na fase de implementação do serviço, na fase de planejamento da adoção, etc.

QP4. Quais são as métricas utilizadas para se medir reusabilidade em SOA?

Objetivo: identificar as métricas que foram utilizadas para se medir reusabilidade em SOA.

\subsection{Processo de Busca}

A estratégia usada para construir os termos de busca seguiu a mesma abordagem utilizada em (Kitchenham et al., 2006). Esta estratégia é sistematizada e define passos para derivar strings de busca a partir de questões de pesquisa, de pontos de vista de experts e de trabalhos relevantes. Foram utilizadas os operadores booleanos OR e AND para incorporar sinônimos e integrar termos das strings de busca que consistiram em SOA ("SOA" / "service oriented architecture" / "SOS" / "service oriented software engineering") e Reuse ("reuse" / "reusability"). As bibliotecas digitais nas quais as buscas automáticas foram realizadas (ACM Digital Library, IEEEXplore e Scopus) foram escolhidas por serem internacionalmente conhecidas no âmbito acadêmico pela qualidade dos estudos indexados. Além disso, estas bibliotecas permitem fácil acesso. Na tabela 2 são listadas as bases de busca e as strings correspondentes.

Tabela 2. Bibliotecas digitais e strings de busca.

\begin{tabular}{|c|l|}
\hline Base de busca & \multicolumn{1}{c|}{ String } \\
\hline $\begin{array}{c}\text { ACM Digital } \\
\text { IEEE Xplore }\end{array}$ & $\begin{array}{l}\text { (Abstract:(("SOA" or "service oriented" or "service-oriented") and } \\
\text { ("reuse" or "reusability" or "reusable"))) or (Title:(("soa" or "service } \\
\text { oriented" or "service-oriented") and ("reuse" or "reusability" or } \\
\text { "reusable"))) }\end{array}$ \\
\hline $\begin{array}{c}\text { ("SOA" OR "service-oriented" OR "service oriented") AND ("reuse" } \\
\text { OR "reusability") }\end{array}$ \\
\hline Scopus & $\begin{array}{l}\text { TITLE-ABS-KEY(("service oriented" OR "service-oriented" OR } \\
\text { "SOA") AND ("reuse" OR "reusability" OR "reusable")) }\end{array}$ \\
\hline
\end{tabular}

\subsection{Critérios de Inclusão e Exclusão}

Com o objetivo de identificar estudos primários relevantes entre as pesquisas de reusabilidade em SOA, foi definido como critério de inclusão estudos primários publicados em conferências e periódicos que discutem como melhorar a reusabilidade em SOA ou em serviços. Como critérios de exclusão foram definidos: i)Trabalhos que apenas citam reuso como benefício de SOA, mas não possuem uma proposta para melhorar ou avaliar a reusabilidade de Arquitetura Orientada à Serviços. ii) Trabalhos que não tratam diretamente sobre como potencializar o reuso em SOA. iii) Trabalhos que tratam a reusabilidade apenas de um software específico. iv) Estudos duplicados (foi considerada a versão mais completa de artigos existentes em mais de um meio de publicação). v) Short papers com menos de três páginas. vi) White papers, relatórios técnicos e livros. vii) Estudos secundários: revisões da literatura, mapeamentos, entre outros. viii) Proposta de doutorado, mestrado, etc. 


\subsection{Condução do Mapeamento Sistemático da Literatura}

O mapeamento foi conduzido entre junho de 2013 e janeiro de 2014. Inicialmente foram executadas buscas automáticas nas bibliotecas digitais utilizando as strings definidas na Tabela 1. Depois foi aplicado o critério de inclusão considerando o título e no resumo dos artigos. Os artigos inclusos tiveram o título, a conferência/jornal e o ano de publicação registrados em uma planilha. Nesta etapa foram incluídos 117 artigos nos quais foram realizadas a leitura do resumo, da introdução, do núcleo da proposta e da conclusão do artigo. Após isso foram aplicados os critérios de exclusão e respondidas as questões de pesquisa definidas. Foram excluídos 87 artigos e incluídos 22. Foram solicitados aos autores via correio eletrônico 6 artigos inacessíveis (Apêndice B) gratuitamente nas bases de busca pesquisadas, no entanto, até o momento da condução não foram obtidas respostas. Todas as etapas do mapeamento foram realizados separadamente por dois pesquisadores. Um terceiro pesquisador analisou e resolveu casos de conflitos.

Para responder as questões de pesquisa, utilizou-se o método keywording, que visou a captura de palavras-chaves para responderem as questões de pesquisa. Com os conflitos resolvidos a lista final dos artigos selecionados para a escrita do relatório de mapeamento sistemático foi analisada para a geração dos resultados.

A fim de calibrar as análises, foi realizado um piloto no qual todos os pesquisadores analisaram independentemente cinco artigos selecionados aleatoriamente e discutiram os resultados por meio de reuniões nas quais um terceiro pesquisador resolveu os conflitos. A análise dos artigos por pesquisadores distintos, a resolução de conflitos por um terceiro pesquisador e a execução de um piloto, visou a diminuição de possíveis vieses da pesquisa.

\section{Resultados}

Nesta seção buscou-se responder as questões de pesquisas definidas em 2.1. O estudo completo poderá ser solicitado aos autores. Na figura 1a, é mostrado o ano de publicação dos 22 artigos resultantes do mapeamento (Apêndice A). 4,5\% (1;22) dos artigos foram publicados respectivamente em 2006 e 2008. Em 2009, foram publicados 13,6\% (3;22) de artigos sobre o tema. Em 2010 houve um aumento na quantidade de publicações, que correspondeu a $27 \%(6 ; 22)$. Esta porcentagem foi repetida em 2012, o que comprova o quanto a investigação sobre Reuso em SOA é atual. Em 2011 foram identificados 9\% $(2 ; 22)$ de artigos referente ao tema. Em 2013, 13,6\% (3;22) dos artigos foram publicados. Considerando que o mapeamento foi realizado entre 2013 e 2014, é possível que artigos em processo de publicação durante este tempo não tenham sido contabilizados. A seleção de artigos entre o período de 2006 e 2013 não foi intencional, apenas correspondeu aos resultados retornados pelas bases de busca. É possível que a preocupação em se propor meios para potencializar o reuso em SOA tenha se iniciado apenas em 2006. 


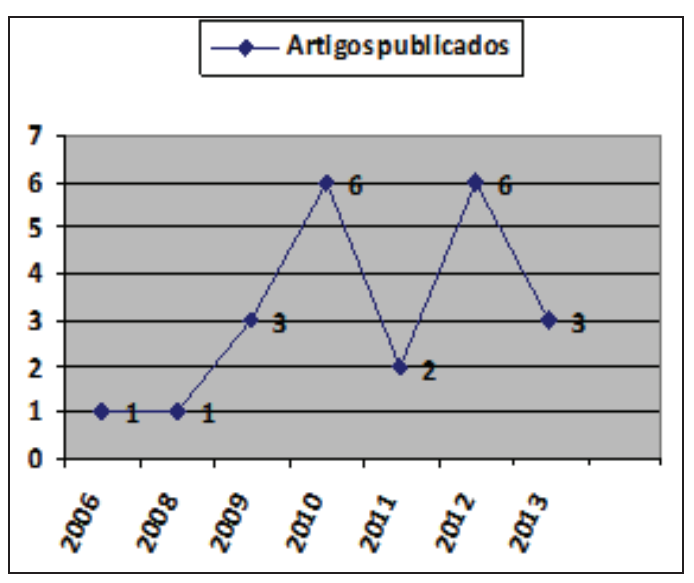

(a)

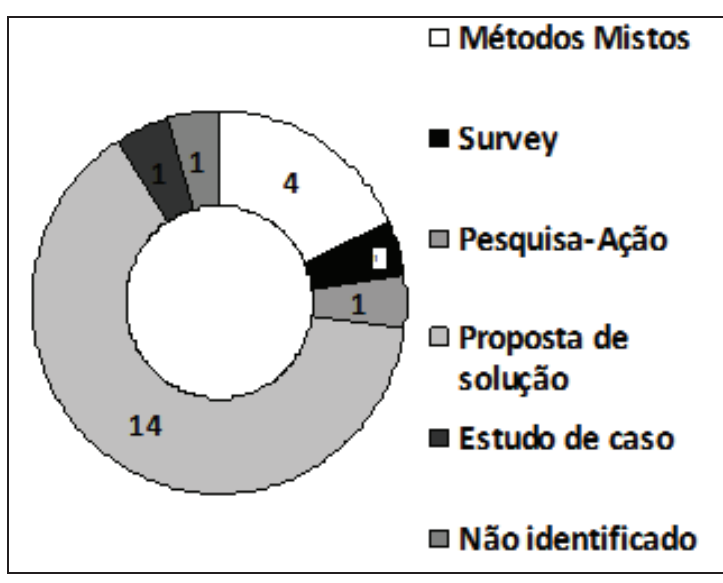

(b)

Figura 1. Quantidade de artigos publicados por ano (1a) e métodos de pesquisa utilizados (1b).

$\mathrm{Na}$ figura $1 \mathrm{~b}$ são exibidos os métodos de pesquisa utilizados nos trabalhos mapeados. 63,6\% $(14 ; 22)$ dos artigos propuseram soluções por meio de metodologias, modelos e abordagens com o propósito de melhorar a reusabilidade em SOA. A elevada porcentagem indica o interesse dos pesquisadores em sugerir meios para potencializar o reuso na Arquitetura Orientada a Serviços. Este fato reafirma a relevância do tema para a pesquisa e para a prática. $18 \%(4 ; 22)$ dos trabalhos utilizaram métodos mistos, dentre os quais $9 \%(2 ; 22)$ corresponderam a proposta e validação de solução por meio de estudo de caso, 4,5\% (1;22) realizaram a mixagem de Pesquisa-Ação com estudo de caso e 4,5\% (1;22) complementaram um survey também com estudo de caso. Apenas um artigo não explicitou o método utilizado.

\subsection{Fatores que influenciam o reuso em SOA}

Consideraram-se fatores quaisquer elementos que influenciam positivamente ou negativamente a reusabilidade em SOA. Foram identificados fatores em 36\% $(8 ; 22)$ dos trabalhos mapeados. 9\% (2;22) dos artigos [P2 e P64] mostraram que o principal fator influenciador do reuso é o custo envolvido no processo. A necessidade de reusar sistemas legados, de integrar ambientes heterogêneos e de maximizar o Retorno do Investimento (ROI) também influencia o reuso em SOA [P37]. A carência de um processo sistemático para identificar e conceber serviços reutilizáveis mostrou ser um fator que influencia o reuso [P86]. Outro fator que influencia a reusabilidade é a busca por processos que possibilitem o desenho de linhas de produtos orientadas a serviços [P58]. O reuso também é influenciado pela necessidade de identificar e gerir a variabilidade dos serviços [P14]. Outros fatores que influenciam o reuso são a busca pela redução de tempo de desenvolvimento de software [P2] e a existência de funcionários com expertise em $\boldsymbol{S O A}$ [P5]. 66\% $(14 ; 22)$ dos artigos não explicitaram claramente fatores que influenciam o reuso em SOA. É perceptível a distinção entre os fatores encontrados nos trabalhos mapeados (Figura 2). Isto comprova que os fatores são subjetivos e variam de acordo com a percepção dos autores sobre o termo. 


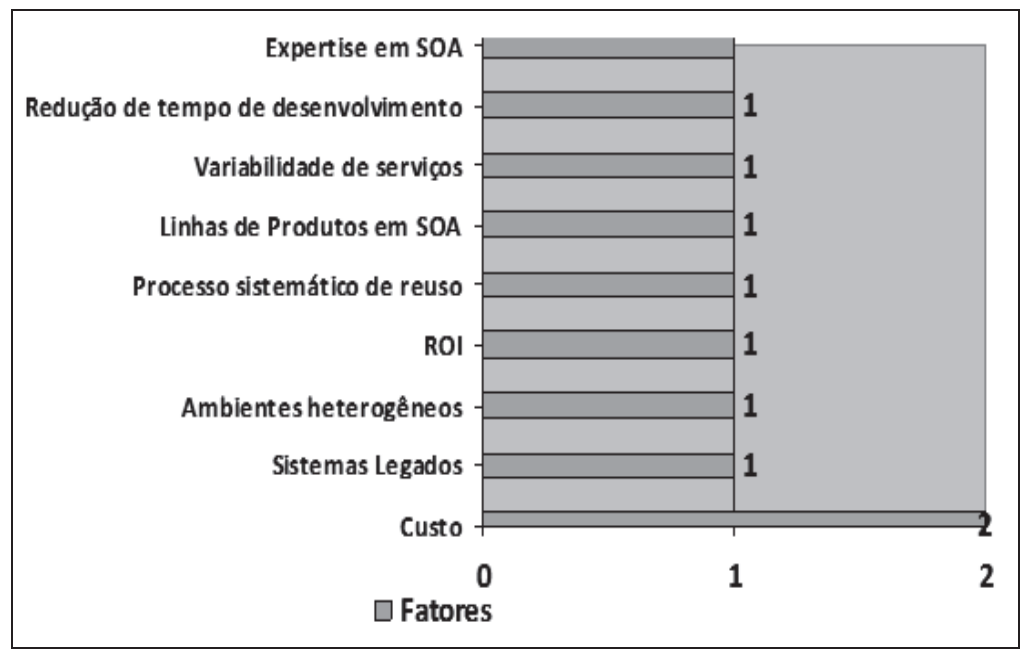

Figura 2. Fatores que influenciam o reuso em SOA.

\subsection{Estratégias adotadas para potencializar o reuso em SOA}

Esta seção visa reportar as estratégias adotadas pelos trabalhos mapeados para tentar potencializar o reuso em SOA. 31,8\% (7;22) dos artigos [P2, P37, P40, P44, P48, P58, P69] apresentaram como estratégia a utilização de conceitos de Linhas de Produtos de Software para o alcance da reusabilidade. Esta foi a estratégia mais representativa. Em $9 \%(2 ; 22)$ dos estudos foi proposta a gestão da variabilidade de serviços [P14, P60]. O uso de repositórios foi adotado na mesma proporção (2;22), [P11, P19]. Outra estratégia adotada foi a gestão da análise e do projeto de serviços, abordada por $18 \%(4 ; 22)$ dos artigos [P6, P67, P86, P74]. Os artigos restantes $(31 \%, 7 ; 22)$ optaram por estratégias distintas que consistiram, distributivamente, em Arquitetura 3D [P1], Governança SOA [P5], gestão da incompatibilidade de serviços [P43], gestão de sistemas legados [P54], Análise de Domínio Orientada a Características (FODA) [P18], Linguagem de Execução de Processos de Negócio (BPEL) com Arquitetura de Componentes de Serviços (SCA) [P110] e Reputação de Serviços [P15].

No [P2] foi proposto o reuso de serviços por meio da Hibridização de Linha de Produtos de Software com SOA (SOPL). Deste modo, os autores propuseram a análise e o projeto de serviços com base nos conceitos de Arquitetura de Linha de Produtos Orientada a Serviços (SOPLA), criando uma mixagem entre as duas áreas para promover a reusabilidade em SOA. Em [P37] foram propostos os conceitos de Linhas de Produtos de Software (SPL) associados aos conceitos de variabilidade para apoiar a descoberta e o registro de serviços com base em 2-LEVEL UDDI. SPL também foram abordados por [P40] na proposição de um Método de Desenvolvimento Orientado à Modelos com a notação BPMN para promover a composição e, consequentemente, o reuso de serviços.

SPL ainda foi utilizada em [P44] para tratar a descoberta, o desenvolvimento e a variabilidade de serviços por meio de um Modelo de Família de Processos de Negócio (BPMF). Este modelo visa auxiliar o design e a implementação de serviços reutilizáveis. No [P48] foi proposta uma junção entre SPL e Modelos de Processos de Negócio em SOA para criar um método de identificação de serviços que une modelos recursos (FMS) de SPLs e Gestão de Processos de Negócios (BPM) em SOA com vista no reuso 
de serviços. SPL também foi utilizada em [P58] na criação de uma abordagem que visa especificar artefatos, definir atividades e fornecer orientações para a equipe de SOA da organização. Esta abordagem promove a utilização destes artefatos em outros projetos, possibilitando o reuso em SOA. Outro registro de SLP foi identificado em [P69] onde foi proposto um modelo de projeto e de desenvolvimento de serviços de domínio.

Em [P19] foi desenvolvido e utilizado um repositório integrado a ERPs e a sistemas legados para promover o reuso em SOA. A ferramenta visou facilitar a gestão, a persistência, o compartilhamento e a busca de serviços e de sistemas disponíveis em um catálogo existente no repositório. No [P11] foi proposto um modelo de referência de análise composto por ativos reutilizáveis existentes em repositórios múltiplos e integráveis nas diferentes camadas de abstração da aplicação. A integração destes repositórios visa promover a gestão e o reuso de artefatos gerados durante a fase de análise de serviços.

$\mathrm{O}$ [P6] propõe uma metodologia para orientar o processo de análise e de projeto de serviços. Para a fase de análise sugeriu-se: i) a identificação do serviço de negócio, ii) a identificação do contexto e iii)a decomposição do serviço de negócio. Para a fase de projeto propôs-se: i) a definição do serviço candidato, ii) a identificação do registro do serviço, iii) a definição do contrato do serviço, iv) a modelagem da rede de serviços, v) a modelagem da coreografia dos serviços. No trabalho citado é proposto que tanto a análise e o projeto de serviços sejam apoiados UML e por SOAML. Em [P86] foi proposto um processo sistemático com base em completude, aplicabilidade, rastreabilidade e precisão para identificar e desenhar serviços reutilizáveis. Este processo é composto por cinco atividades: i) Identificar processos de negócio; ii) Especificar processos de negócio, iii) Identificar serviços reusáveis; iv) Especificar serviços reusáveis e v) Projetar serviços reusáveis. Em [P74] propôs-se uma abordagem de projeto de serviços orientada a padrões que consiste em três fases: i) análise de negócios, ii) modelagem de serviços e iv) desenvolvimento de componentes de software.

No [P14] o conceito da variabilidade de serviços foi utilizado na construção de uma metodologia para identificar e modelar possíveis variâncias em SOA. Para permitir a representação da variabilidade foi proposto o uso da extensão varWSDL na geração de documentos WSDL, XML e na especificação de artefatos de serviços. A variabilidade também foi utilizada em [P60] que propôs uma Metodologia baseada em Engenharia Orientada a Variação para identificar e controlar variâncias de serviços. Para tal foi utilizada também uma técnica denominada Design de Serviço Orientado à Variação (VOSD) para descobrir e derivar automaticamente variâncias de serviços a partir de um repositório reutilizável.

No [P18] foi utilizado o conceito de Análise de Domínio Orientada à Características (FODA) na proposição de um método para guiar a análise e o projeto de serviços reutilizáveis. A proposta sugere a análise do contexto, dos requisitos do domínio e das aplicações para extrair características de serviços que poderão ser reusados em uma SOA. Em [P54] foi proposta a análise da reutilização de sistemas legados em arquiteturas SOA com base em Engenharia Orientada a Modelo(MDE) e Linguagens Específicas do Domínio (DSL) para representar e analisar serviços candidatos em SOA integráveis a sistemas legados. 
Em [P110] utilizou-se a combinação de Linguagem de Execução de Processos de Negócio (BPEL4WS) e Arquitetura de Componentes de Serviços (SCA) para a criação de padrões de aplicações reutilizáveis com o uso de EJB e JCA. No [P43] padrões adaptadores com base em gestão da incompatibilidade foram propostos para identificar e evitar casos de incompatibilidade na descoberta de serviços.

Em [P1] os autores propuseram o uso de Arquitetura 3D (Decisão - Decision, Níveis - Degree e Dados - Data) para a Automação de Serviços. Com base nesta arquitetura foi desenvolvido um sistema denominado SDQA para apoiar o projeto e a implementação de serviços reutilizáveis.

Em [P5] a Governança em SOA inspirou a criação de um modelo de reuso com modularidade e sem modularidade baseada nesta abordagem.

A eficiência de cada estratégia pode ser influenciada por diversos fatores como ambiente organizacional, conhecimento da equipe em SOA, tecnologias, softwares, método de aplicação, objetivos, entre outros. Portanto, só é possível avaliar qual estratégia melhor potencializa o reuso mediante a real adoção da proposta. A variedade de estratégias encontradas nos artigos mapeados possibilita a escolha daquela que mais se adequa aos propósitos de quem busca reutilizar serviços em SOA. Porém, a ampla utilização de Linhas de Produtos de Software $(36 \%, 8 ; 22)$ indica que os conceitos desta área podem prover um efetivo meio para alcançar o reuso. Nesse contexto os artigos [P37] e [P44] se destacaram por validar a proposta baseada em LPS através da condução de um estudo de caso e por reportar os resultados.

\subsection{Fase do ciclo de vida em que a estratégia foi utilizada}

$45 \%(10 ; 22)$ dos artigos utilizaram a estratégia proposta na fase de análise [P2, P6, P11, P18, P37, P40, P43, P44, P69, P86]. Esta fase engloba atividades de elicitação e de especificação de requisitos de serviços. Em 50\% $(11,22)$ dos trabalhos mapeados a estratégia foi utilizada na fase de projeto, que envolve a modelagem dos serviços [P2, P6, P15, P19, P40, P43, P44, P58, P60, P74, P86]. A fase de desenvolvimento foi abordada em 22\% (5; 22) dos artigos [P2, P14, P37, P58, P110]. No P37 a estratégia foi utilizada na fase de implantação. Em $4,5 \%(1 ; 22)$ dos artigos a estratégia foi tratada na fase de automação [P1].

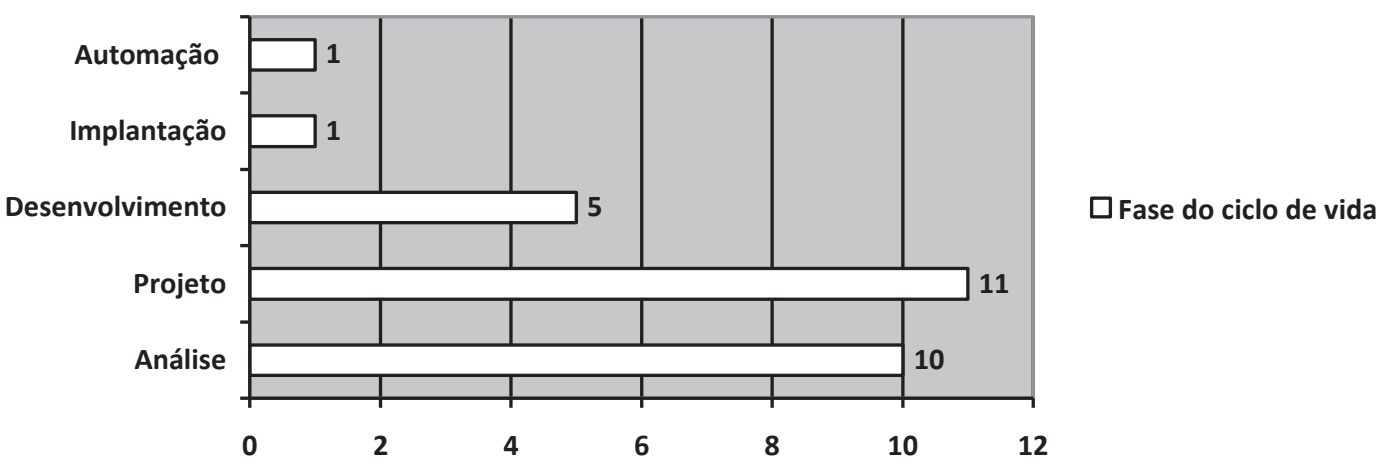

Figura 3. Fases do ciclo de vida em que a estratégia é utilizada. 
Foi observado que apenas dois artigos focaram respectivamente a fase de implantação e de automação. Nenhum dos artigos incluídos propôs melhorias que promovam o reuso na fase de monitoramento de serviços. Estas constatações indicam que a área de SOA é academicamente carente de trabalhos que potencializem a reusabilidade nestas fases. Esta lacuna favorece a realização de pesquisas que proponham meios para possibilitar que componentes de Arquitetura Orientada a Serviços sejam reutilizados nas fases anteriormente citadas.

\subsection{Métricas}

Buscou-se identificar possíveis métricas utilizadas para medir a reusabilidade em SOA. Os artigos incluídos no mapeamento não explicitaram indicadores de medição. Isso se justifica porque todos os trabalhos propuseram melhorias para a fase de análise, projeto e desenvolvimento, não incluindo a fase de monitoramento, na qual normalmente os serviços são mensurados. Houve uma exceção em 3 dos artigos excluídos [P33, P39, P64] (Apêndice C), que respectivamente propuseram métricas para mensurar o nível de dependência de serviços, o nível de acoplamento dos serviços e o nível de incompatibilidade de reuso de um serviço. No entanto, estes artigos não tratavam diretamente como potencializar o reuso em SOA, sendo excluídos com base no segundo critério de exclusão definido na seção 2.3.

\section{Discussão}

Apesar da quantidade relevante de trabalhos que propõem melhorias para potencializar o reuso e da diversidade de estratégias utilizadas, é perceptível a carência da aplicação real das propostas. Neste sentido, dos 22 artigos mapeados, dois [P37, P44] se destacaram pela completude, consistência e por apresentarem a validação das metodologias criadas por meio de estudos de caso. Porém, até o estudado, os trabalhos restantes não indicam que a proposta foi validada. Há carência também quanto a identificação de fatores que influenciam o reuso em SOA, visto que $66 \%$ dos artigos não explicitam este elemento. Referente as fases do ciclo de vida, foi identificado que as fases de implementação, automação e monitoramento de SOA são escassas de métodos que potencializem o reuso. A ausência, até o estudado, de trabalhos que definam métricas para mensurar e melhorar o reuso também consiste em uma lacuna na reusabilidade em SOA. Neste sentido, a área apresenta uma rica variedade de assuntos a serem investigados por pesquisadores que possuem interesse no tema.

\section{Trabalhos relacionados}

Uma investigação semelhante foi realizada por Daud e Wan Kadir (2012) que realizaram um Mapeamento Sistemático da Literatura com o propósito de avaliar os atributos de qualidade de SOA. No entanto Daud e Wan Kadir (2012) não focam o reuso em especial. Shanmugasundaram et al. (2012) também apresentou um trabalho similar no qual avaliou oportunidades de pesquisa relacionadas a reuso em SOA por meio de uma revisão da literatura. No entanto, os autores não citam fatores, estratégias, fases do ciclo de vida e métricas relacionados ao reuso. Portanto, estes pontos consistem em um diferencial do presente estudo, sendo relevante para pesquisadores e organizações que visam se aprofundar sobre os aspectos da reusabilidade em SOA. 


\section{Considerações finais}

Este artigo apresentou um Mapeamento Sistemático da Literatura realizado entre junho de 2013 e janeiro de 2014 com o propósito de identificar: o estado da arte neste contexto; os fatores que influenciam positivamente e negativamente a reusabilidade, as estratégias comumente adotadas para a melhoria contínua do reuso, as fases do ciclo de vida em que as estratégias adotadas foram utilizadas e as métricas associadas a mensuração desse atributo.A pesquisa foi motivada pela ausência, até o estudado, de um estudo sistemático que agregue aspectos especificamente relacionados a reuso em SOA.

O estudo possui por principal limitação o viés decorrente da interpretação dos autores na análise dos artigos, que pode ter prejudicado a inclusão de trabalhos relevantes. Buscou-se atenuar este viés por meio da condução separadamente do mapeamento por dois pesquisadores e da avaliação das análises realizadas por um terceiro pesquisador. Os termos utilizados nas strings de busca também podem ter afetado a quantidade de artigos retornados, consequentemente, artigos primários importantes podem não ter sido analisados. Entretanto procurou-se mitigar este riso através da calibração das strings de busca utilizadas.

Como a investigação sobre reuso em SOA no presente trabalho englobou apenas artigos acadêmicos, é sugerido que as questões de pesquisa definidas na seção 2.1. sejam respondidas por meio de um estudo empírico que investigue fatores, estratégias e métricas utilizadas para potencializar o reuso em SOA na prática. O estudo poderá ser realizado por meio de estudo de caso ou survey em organizações reais. O resultado possibilitará uma análise comparativa da reusabilidade em SOA na academia e na indústria.

\section{Referências}

Alferez, G. H. e Pelechano, V. (2011) "Systematic reuse of web services through software product line engineering". 9th IEEE European Conference on Web Services (ECOWS).

Budgen, D. (2008) Turner, Mark; Brereton, Pearl; and Kitchenham, Barbara. "Using Mapping Studies in Software Engineering", Proceedings of Psychology of Programming Interest Group (PPIG), Lancaster University, pp. 195-204.

Dan, A., Johnson, R. D. e Carrato, T. (2008) "SOA service reuse by design". 2nd international workshop on Systems development in SOA environments.

Daud, N. M. e Wan Kadir, W. M. N. (2012) Systematic mapping study of quality attributes measurement in service oriented architecture. In: Proceedings - ICIDT 2012. IEEE, New York, USA, pp. 626-631

Dias, J., Oliveira, J., Meira, S. (2012) "Pontos Chaves para Adoção de Uma Arquitetura Orientada a Serviços: Uma Análise Comparativa de Modelos de Maturidade SOA da Indústria”. Simpósio Brasileiro de Sistemas de Informação, São Paulo.

Dias, J., Oliveira, J., Meira, S. (2013) "Estudo Empírico sobre Adoção de SOA: Um Mapeamento Sistemático da Literatura". Simpósio Brasileiro de Qualidade de Software, Bahia. 
Erl, T. (2005) Service Oriented Architecture: Concepts, Technology and Design. Prentice-Hall, Englewood Cliffs.

Brahe, S. BPM on top of SOA: Experiences from the financial industry (2007) Lecture Notes in Computer Science, 4714 LNCS, pp. 96-111.

Germ'n H. Alférez, Vicente Pelechano. (2011) "Systematic Reuse of Web Services through Software Product Line Engineering," ecows, pp.192-199, 2011 IEEE Ninth European Conference on Web Services.

Karthikeyan T. e Geetha J. (2013) A Tool for Measuring SOA Service Granularity. IJCA Special Issue on International Conference on Communication, Computing and Information Technology ICCCMIT(3):17-21.

Josuttis, N. (2007) "SOA in Practice - The Art of Distributed System Design.” O'Reilly Media.

Kitchenham, B. A. (2007) "Guidelines for performing Systematic Literature Reviews in Software Engineering”. In: EBSE Technical Report.

Kitchenham, Barbara; Mendes, Emiliaand Travassos, Guilherme. (2006). "A systematic review of cross vs within company cost estimation studies", Proceedings EASE, 2006, pp. 89-98.

La, H. J. e Kim, S. D. (2009) Adapter patterns for resolving mismatches in service discovery. Proceedings of International Conference on Service-Oriented Computing.

Petersen, Kai; Feldt, Robert; Mujtaba, Shahid e Mattsson, Michael. (2008) "Systematic Mapping Studies in Software Engineering",12th International Conference on Evaluation and Assessment in Software Engineering (EASE), University of Bari, Italy, 26 - 27 June.

Ponnalagu, k., Nanjangud C., e Gangadharan, G. R. (2011) "Integrated asset analysis framework for model-driven development of SOA based solutions". ICSOC Workshops, volume 7221 of Lecture Notes in Computer Science, page 257269. Springer,

Shanmugasundaram, G. Prasanna Venaktesan, V. Punitha Devi, C. (2013) "Modeling Measures for Service Interpretation in Discoverability of Service Oriented Architecture" Procedia - Social and Behavioral Sciences, Volume 73, 27 February, Pages 128-135, ISSN 1877-0428.

Yeom, K., Park, J. e Moon, M (2011) "Registry and discovery of services with variability based on 2-level UDDI". 9th IEEE International Symposium on Parallel and Distributed Processing with Applications Workshops (ISPAW). Maio.

\section{Apêndice A}

\begin{tabular}{|l|l|}
\hline \multicolumn{1}{|c|}{ ID } & \multicolumn{1}{c|}{ Artigo incluído } \\
\hline P1 & 3D architecture viewpoints on service automation \\
\hline P2 & An approach for e-Commerce on-demand service-oriented product line development \\
\hline P5 & The influence of SOA governance mechanisms on IT flexibility and service reuse \\
\hline P6 & A pragmatic approach for analysis and design of service inventories \\
\hline P11 & Integrated asset analysis framework for model-driven development of SOA based \\
\hline
\end{tabular}




\begin{tabular}{|l|l|} 
& solutions \\
\hline P14 & Promoting reuse in web services by managing variability \\
\hline P15 & $\begin{array}{l}\text { ReputationNet: A Reputation Engine to Enhance ServiceMap by Recommending } \\
\text { Trusted Services }\end{array}$ \\
\hline Service-oriented domain analysis method \\
\hline P19 & $\begin{array}{l}\text { The implementation experience of an advanced service repository for supporting } \\
\text { service-oriented architecture }\end{array}$ \\
\hline P40 & Registry and discovery of services with variability based on 2-level UDDI \\
\hline P43 & Adapter patterns for resolving mismatches in service discovery \\
\hline P44 & $\begin{array}{l}\text { An approach to developing reusable domain services for service oriented } \\
\text { applications }\end{array}$ \\
\hline P48 & $\begin{array}{l}\text { Bridging Software Product Lines and Service-Oriented Architectures for Service } \\
\text { Identification Using BPM and FM }\end{array}$ \\
\hline P54 & Reusing legacy systems in a service-oriented architecture: A model-based analysis \\
\hline P58 & SOPLE-DE: an approach to design service-oriented product line architectures \\
\hline P60 & Towards a Variability Model for SOA-Based Solutions \\
\hline P67 & A service lifecycle and information model for service-oriented architectures \\
\hline P69 & An approach to enhancing reusabilities in service development \\
\hline P74 & Design Web services: Towards service reuse at the design level \\
\hline P86 & A Formal Approach to Devising a Practical Method for Modeling Reusable Services \\
\hline P110 & $\begin{array}{l}\text { Building business processes or assembling service components: Reuse services with } \\
\text { BPEL4WS and SCA }\end{array}$ \\
\hline
\end{tabular}

\section{Apêndice B}

\begin{tabular}{|l|l|}
\hline \multicolumn{1}{|c|}{ ID } & \multicolumn{1}{c|}{ Artigo inacessível } \\
\hline p4 & $\begin{array}{l}\text { Anti-pattern free code-first web services for state-of-the-art Java WSDL generation } \\
\text { tools }\end{array}$ \\
\hline p20 & $\begin{array}{l}\text { The study on software reuse based on service composition and dependency-aware } \\
\text { service oriented architecture }\end{array}$ \\
\hline p22 & Two-level service-oriented architecture based on product-line \\
\hline p34 & Modified MVC-design patterns for service oriented applications \\
\hline p59 & Towards a user-oriented framework for web service discovery, reuse and evolution \\
\hline p108 & What COTS and software reuse teach us about SOA \\
\hline
\end{tabular}

Apêndice $\mathrm{C}$

\begin{tabular}{|c|l|}
\hline ID & \multicolumn{1}{|c|}{ Artigos excluídos que abordam métricas de reuso em SOA } \\
\hline P33 & Measuring the Conceptual Coupling of Services Using Latent Semantic Indexing \\
\hline P39 & Simple metric for assessing quality of service design \\
\hline P64 & A metric for composite service reusability analysis \\
\hline
\end{tabular}

